\title{
Spin Polarization and Exchange Interaction in a Diluted Magnetic Qunautm Dot
}

\author{
A. John Peter ${ }^{1}$ and K. Lily Mary Eucharista ${ }^{2}$ \\ ${ }^{1}$ Government Arts College, Melur, Madurai-625 106, India \\ ${ }^{2}$ Arul Anandar College, Karumathur, Madurai-625 514, India \\ Correspondence should be addressed to A. John Peter, a_johnpeter@rediffmail.com
}

Received 15 October 2008; Revised 20 February 2009; Accepted 1 April 2009

Recommended by Joseph Poon

The spin interaction energy of different $\mathrm{Mn}^{2+}$ ions with and without an itinerant electron is evaluated for different dot radii. Magnetization is calculated for various concentrations of $\mathrm{Mn}^{2+}$ ions with different dot sizes. Spin polaronic shifts are estimated using a mean field theory. The lowest binding energies of electrons in a $\mathrm{Cd}_{1-x} \mathrm{Mn}_{x} \mathrm{Te}$ quantum dot are also calculated. Results are obtained for $\mathrm{Cd}_{1-x_{\text {in }}} \mathrm{Mn}_{x_{\text {in }}} \mathrm{Te} / \mathrm{Cd}_{1-x_{\text {out }}} \mathrm{Mn}_{x_{\text {out }}} \mathrm{Te}$ structures as a function of the dot radius variationally. It is found that (i) more number of $\mathrm{Mn}^{2+}$ spins enhance the spin polaronic effect and it varies linearly with the concentration, (ii) spin polarization of $\mathrm{Mn}^{2+}$ ions increases with the concentration for any dot radii, (iii) the magnetization of Mn subsystem increases with the concentration of $\mathrm{Mn}^{2+}$ ions and this feature is predominant for smaller dots, and (iv) variation of increase in ionization energy is sharper for smaller dots with increase in concentration. These results are discussed with the available data in literature.

Copyright (C) 2009 A. J. Peter and K. L. M. Eucharista. This is an open access article distributed under the Creative Commons Attribution License, which permits unrestricted use, distribution, and reproduction in any medium, provided the original work is properly cited.

\section{Introduction}

Recent advances in nanofabrication technology have allowed the construction of structures with lower dimensions. The most recent achievement is the fabrication of zero-dimension quantum structures, usually called quantum dots. Due to their small size these structures exhibit physical properties that are quite different from those of the bulk semiconductor constituents. It is expected that these properties will show more pronounced differences as the confinement is increased with the lowering of the dimensionality. More specifically, the shape and the size of the nanostructure have a strong influence on the optical properties [1].

Diluted magnetic semiconductors (DMSs) are expected to play an important role in interdisciplinary materials science and future electronics because charge and spin degrees of freedom accommodated into a single material exhibit interesting magnetic, magneto-optical, magnetoelectronic, and other properties [2]. Controlling the spin state of electrons provides an important versatility for future nanoelectronics [3]. Most of the envisioned spintronic devices are based on spin transfer mechanisms on the nanoscale.
For this purpose, new materials have been synthesized with highly spin polarized bands, and novel experimental techniques are being applied to characterize the spin state of the charge carriers [4-6]. The range, strength, and sign of exchange interactions between magnetic impurities in DMSs depend on the density and nature of the states at the Fermi level. Mn-doped semiconductors of the families (II,Mn)-VI and (III,Mn)-V order ferromagnetically in the presence of carriers that mediate indirect exchange interactions between Mn. (III,Mn)V DMSs are promising spintronic materials with high spin polarization of bound magnetic polarons [7-9] and with a wide variety of spin-dependent transport properties [10]. While considerable effort is concentrated to enhance the ferromagnetic transition temperature $[11,12]$. Smith et al., [13] showed the evidence of exciton magnetic polarons in CdMnTe QDs form through the spontaneous ferromagnetic alignment of the Mn.

Study of DMSs and their heterostructures have centered mostly on II-VI semiconductors, such as CdTe and ZnSe, in which the valence of the cations matches that of the common magnetic ions such as Mn. Although this phenomenon makes these DMSs relatively easy to prepare in bulk form 
as well as in thin epitaxial layers, II-VI-based DMSs have been difficult to dope to create $\mathrm{p}$ and n-type, which made the material less attractive for applications. Moreover, the magnetic interaction in II-VI DMSs is dominated by the antiferromagnetic exchange among the Mn spins, which results in the paramagnetic, antiferromagnetic, or spin glass behavior of the material. It was not possible until very recently to make a II-VI DMS ferromagnetic at low temperature in modulation-doped QW structures exploiting RKKY mechanism [14].

Diluted magnetic semiconductors (DMSs) are compound of alloy semiconductors containing a large fraction of magnetic ions $\left(\mathrm{Mn}^{+2}, \mathrm{Cr}^{+2}, \mathrm{Fe}^{+2}, \mathrm{Co}^{+2}\right)$ and are studied mainly on II-VI-based materials such as CdTe and ZnSe. This is because such +2 magnetic ions are easily incorporated into the host II-VI crystals by replacing group II cations. In such II-VI-based DMSs such as (CdMn)Te, (CdMn)Se, magneto-optic properties were extensively studied, and optical isolators were recently fabricated using their large Faraday effect [15]. Also, recently, a controllable fabrication of dots with only a single $\mathrm{Mn}$ ion and the photoluminescence studies distinguishing various positions of such Mn ion or anisotropy in the quantum confinement and the temperature of the onset of magnetization in DMS QDs higher than in the bulk DMSs have been studied [16, 17]. Moreover, the carriermediated ferromagnetism bulk DMS such as light- and bias-controlled ferromagnetism have also been studied. The interaction among these spins leads to ferromagnetic order at low temperatures, which is not only to create spin-polarized carriers but also optical or electrical injection can create highly spin-polarized carrier density even in nonmagnetic semiconductors [18]. Petukhov et al. [19], have proposed a model of carrier-mediated ferromagnetism in semiconductors accounting for the temperature dependence of the carriers. Their model permits analysis of the thermodynamic stability of competing magnetic states, opening the door to the construction of magnetic phase diagrams. The complete details of properties of DMS material have been reviewed by Furdyna [20]. Optically detected cyclotron resonance of two-dimensional electrons has been studied in nominally undoped $\mathrm{CdTe} /(\mathrm{Cd}, \mathrm{Mn}) \mathrm{Te}$ quantum wells [21] where they studied an increase of the electron cyclotron mass from $0.099 \mathrm{~m}_{0}$ to $0.112 \mathrm{~m}_{0}$ with well width decreasing from 30 down to $3.6 \mathrm{~nm}$. Diluted magnetic oxide semiconductors are considered to be one of the strong candidates to realize room temperature ferromagnetism. Among them, $\mathrm{Mn}$-doped $\mathrm{ZnO}$ can be regarded as a class of $\mathrm{Mn}$-doped II-VI semiconductors, and the properties are similar to the typical II-VI magnetic semiconductors [22, 23].

In this work, the lowest binding energy of the donor electron in a diluted magnetic semiconductor of a $\mathrm{Cd}_{1-x} \mathrm{Mn}_{x} \mathrm{Te}$ quantum dot is calculated. We investigate theoretically the donor bound spin polaronic effect in a quantum dot. The mean field theory with modified Brillouin function that is already used for bulk and quantum well cases has been extended to the case of a QD, and we estimate the spin polaronic shifts to the impurity ionization energies. Moreover, the introduction of magnetic ions such as Mn into these compounds leads to the formation of diluted magnetic semiconductors, in which the exchange interaction between the magnetic ions and electronic states opens perspectives for interesting new phenomena. One such possibility is observing a situation in which the exchange-interactionenhanced spin splitting of a Landau level coinciding with the energy separation between adjacent Landau levels (cyclotron energy), where interaction of the two resonances might be expected. Donor ionization energy and the spin interaction energy are calculated with different concentrations of $\mathrm{Mn}^{2+}$ ions for different dot radii. The spin interaction energy of among $\mathrm{Mn}^{2+}$ ions is evaluated for different dot radii. Magnetization is calculated for various concentrations with the dot sizes.

We would like to point out that the lowest binding energy and the impurity binding are calculated for a $\mathrm{Cd}_{1-x} \mathrm{Mn}_{x} \mathrm{Te}$, within the single band approximation, varying the variational parameter. The magnetization is computed using the variational parameter with the impurity concentration. The donor electron in a doped semimagnetic semiconductor has a huge orbit $(\sim 50 \AA)$ in which there occur several manganese ions which are polarized due to the spin of the carrier which is an exchange mechanism resulting the concept of Bound Magnetic Polaron (BMP). The spin polaronic effect is also computed with the same variational parameter and the wave function, and it is estimated with the mean field approximation using modified Brillouin function.

\section{Model and Calculations}

2.1. Ionization Energy. Our system consists of a spherical dot (depth $\mathrm{V}$, and radius $\mathrm{R}$ ) containing a donor impurity inside the QD of the magnetically nonuniform "spin-doping" superlattice system such as $\mathrm{Cd}_{1-x_{\text {in }}} \mathrm{Mn}_{x_{\text {in }}} \mathrm{Te} / \mathrm{Cd}_{1-x_{\text {out }}} \mathrm{Mn}_{x_{\text {out }}} \mathrm{Te}$ with different concentrations of $x_{\text {in }}$ and $x_{\text {out }}$ of $\mathrm{Mn}$ ions in- and outside the QD. Such a QD may be fabricated by the method of evolution of self-assembled quantum dots (QDs) in the Stransky-Krastanow mode as in the case of $\mathrm{Cd}_{1-x_{\text {in }}} \mathrm{Mn}_{x_{\text {in }}}$ Se QDs or by electron beam lithography and wet chemical etching which is used to fabricate quantum wires [24].

The system is described by the following Hamiltonian:

$$
H=H_{e}+g \mu_{B} s_{z} B-J_{\mathrm{s}-\mathrm{d}} \sum_{I} \bar{S}_{I} \bullet \bar{s} \delta\left(\bar{r}-\bar{R}_{I}\right) .
$$

Let us analyze the three terms contributing to $H . H_{e}$ is the part of Hamiltonian which describes the itinerant electron. The second term is the Zeeman coupling between localized spins and an external magnetic field $B$ where $\mu_{B}$ is the Bohr magneton. And the third term is the magnetic Hamiltonian for one itinerant electron with spin $\bar{s}$ located at $\bar{r}$ and one $\mathrm{Mn}$ ion with $\bar{S}$ located at $\bar{R}$. In the presence of hydrogenic impurity, the Hamiltonian is the sum of kinetic energy and potential energy, given by

$$
H_{e}=-\frac{\hbar^{2}}{2 m^{*}} \nabla^{2}-\frac{e^{2}}{\varepsilon_{0} r}+V(r),
$$

where $m^{*}$ is the effective mass, and $\varepsilon_{0}$ is the static dielectric constant of CdTe. By introducing the effective Rydberg $R_{y}^{*}$, 
as the unit of energy and the effective Bohr radius (60 $\AA$ ) as the length unit, the Hamiltonian given in (2) becomes

$$
H_{e}=-\nabla^{2}-\frac{2}{r}+\frac{V(r)}{R_{\mathbf{y}}^{*}} .
$$

The lowest state energies are obtained using the wave function

$$
\psi= \begin{cases}N_{2} \frac{\sin k_{1} r}{r}, & r \leq R \\ N_{3} \exp \left(-k_{2} r\right), & r>R\end{cases}
$$

where $N_{2}, N_{3}$ are normalization constants, $k_{1}=$ $\left(2 m^{*} E / \hbar^{2}\right)^{1 / 2}$, and $k_{2}=\left(2 m^{*}(E-V) / \hbar^{2}\right)^{1 / 2}$. For a finite barrier case we choose $x=0.02$; hence $V=29.92 R_{y}^{*}$.

With the inclusion of the impurity potential in the Hamiltonian forces to use of the variational approach. Then the trial wave function for the ground state with the impurity present is taken as

$$
\psi= \begin{cases}N_{4} \frac{\sin k_{1} r}{r} \exp \left(-\alpha_{1} r\right), & r \leq R \\ N_{5} \exp \left(-k_{2} r\right) \exp \left(-\alpha_{1} r\right), & r>R\end{cases}
$$

where $\alpha_{1}$ is the variational parameter, and $N_{4}, N_{5}$ are normalization constants.

The ionization energy is given by

$$
E_{\text {ion }}=E_{\mathrm{sub}}-\langle H\rangle_{\min },
$$

where $E_{\text {sub }}$ is the lowest subband energy obtained with the impurity. Thus the ionization energy is obtained, varying $\alpha_{1}$ for different dot sizes with different concentrations of $\mathrm{Mn}$ ions.

The $H_{\text {zee }}$ term is given by

$$
H_{\text {zee }}=g \mu_{B} s_{z} B=x J_{\mathrm{s}-\mathrm{d}} s_{z}\left\langle S_{z}\right\rangle \text {, }
$$

which is the Zeeman coupling between localized spins and an external magnetic field $B$. Direct interactions between the magnetic moments of $\mathrm{Mn}$ ions are much smaller than the interaction with the carrier spins [25], and also for an electron spin, the separation between the Zeeman levels is given by $\hbar \omega=\hbar \gamma B_{0}$ where $\gamma$ is the gyromagnetic ratio. For $B_{0} \sim 40$ Tesla, we obtain a value of $\sim 5 \mathrm{meV}$ for the separation. As this is small when compared to the exchange energy, we drop the Zeeman Effect. Equation (7) is the antiferromagnetic exchange interaction arising between the spin of a conduction electron and the $\mathrm{Mn}^{2+}$ spins. Here $S_{I}$ is the spin of the $\mathrm{Mn}^{2+}$ ion at position $R_{I}$, and $s$ is the spin of the conduction electron centered at $r$. The exchange interaction $J\left(r, R_{I}\right)$ is dependent on the overlap between the orbital of the conduction electron and of the $3 \mathrm{~d}$ electrons.

2.2. Spin Polaronic Effect. Kasuya and Yanase [26], who explained the transport properties of magnetic semiconductors, originally developed the theory of spin polaron (SP). This mean field theory, which invokes the exchange interaction between the carrier and magnetic impurity in the presence of an external magnetic field $B$, yields the spin polaronic shift, $E_{\mathrm{sp}}$, with the modified Brillouin function [27]:

$$
E_{\mathrm{sp}}=\frac{1}{2} \alpha N_{0} \int x S_{0}(x)|\psi|^{2} B_{s}\left[\frac{S \alpha|\psi|^{2}}{2 k_{B}\left[T+T_{0}(x)\right]}\right] d \tau,
$$

where $\alpha$ is the exchange coupling parameter, $S$ is the $\mathrm{Mn}^{2+}$ spin, and $x N_{o}$ is the $\mathrm{Mn}$ ion concentration. The integration is on spatial coordinates. Also $g \approx 2, S_{0}(x)$, the effective spin, and $T_{0}(x)$, the effective temperature, are the semi-phenomenological parameters, which describe the paramagnetic response of the $\mathrm{Mn}^{2+}$ ions in the bulk $\mathrm{Cd}_{1-x} \mathrm{Mn}_{x} \mathrm{Te}$ [28], $k_{B}$ is the Boltzmann constant, and $B_{s}(\eta)$ is the modified Brillouin function. In (8), $\psi$ is the envelope function given by (5).

The parameters used in our calculations are $N_{0}=$ $2.94 \times 10^{22} \mathrm{~cm}^{-3}, \alpha N_{0} \approx 220 \mathrm{mev}$, and the semiphenomenological parameters $S_{o}\left(X_{\text {in }}=0.02\right)=1.97$, $T_{o}\left(X_{\text {in }}=0.02\right)=0.94,[29]$. The lattice constant is around $6.48 \AA$. We estimate $N_{0}$, the atomic concentration of $\mathrm{Cd}$, to be $2.940 \times 10^{22} \mathrm{~cm}^{-3}$. For $x=0.01$ in $\mathrm{Cd}_{1-x_{\text {out }}} \mathrm{Mn}_{x_{\text {out }}} \mathrm{Te}$ barrier, there are about $134 \mathrm{Mn}^{2+}$ ions in a volume $4 \pi a^{* 3} / 3$.

Using the envelop function given in (4) with the appropriate variational parameters, we obtain

$$
E_{\text {sp }}=\frac{1}{2} \alpha N_{0}\left[X_{\text {in }} S_{0}\left(X_{\text {in }}\right) I_{1}+X_{\text {out }} S_{0}\left(X_{\text {out }}\right) I_{2}\right],
$$

where

$$
\begin{gathered}
I_{1}=\int\left|\psi_{\text {in }}\right|^{2} B_{s}\left(\eta_{1}\right) d \tau, \quad I_{2}=\int\left|\psi_{\text {iout }}\right|^{2} B_{s}\left(\eta_{2}\right) d \tau, \\
B_{s}\left(\eta_{j}\right)=\frac{2 S+1}{2 S} \operatorname{coth}\left(\frac{2 S+1}{2 S} \eta_{j}\right)-\frac{1}{2 S} \operatorname{coth}\left(\frac{\eta_{j}}{2 S}\right),
\end{gathered}
$$

with $\eta_{1}=S \alpha\left|\psi_{\text {in }}\right|^{2} / 2 k_{B}\left[T+T_{0}\left(x_{\text {in }}\right)\right]$ and $\eta_{2}=S \alpha\left|\psi_{\text {out }}\right|^{2} /$ $2 k_{B}\left[T+T_{0}\left(x_{\text {out }}\right)\right]$.

The factor $B_{s}\left(\eta_{j}\right)$ represents the spin polarization of the $\mathrm{Mn}^{+2}$ cations. The spin of the $\mathrm{Mn}^{+2}$ cation is $S=5 / 2$. $B_{s}\left(\eta_{j}\right)$ is the standard Brillouin function. Such a simplified Brillouin-function approach is quite common when dealing with quasi-low dimensional systems.

2.3. Spin Exchange-Interaction Energy. The magnetization of the magnetic ions competes with spin-orbit coupling [30, 31]:

$$
\xi=\frac{g_{\mathrm{Mn}} \mu_{B} S B-J_{s-d} S\left(n_{\mathrm{down}}-n_{\mathrm{up}}\right) / 2}{k_{B} T},
$$

where $\mu_{B}$ is the Bohr magneton, $g_{\mathrm{Mn}}$ is the $g$ factor of $\mathrm{Mn}$ ions, and $n_{\text {down }}$ and $n_{\text {up }}$ are the spin-down and spin-up concentrations measured for a particular dot radius. The first term in the numerator of (11) represents the contribution of the Zeeman coupling between the localized spin and the magnetic field. The second term in the numerator of (11) (sometimes called "feedback mechanism") represents 


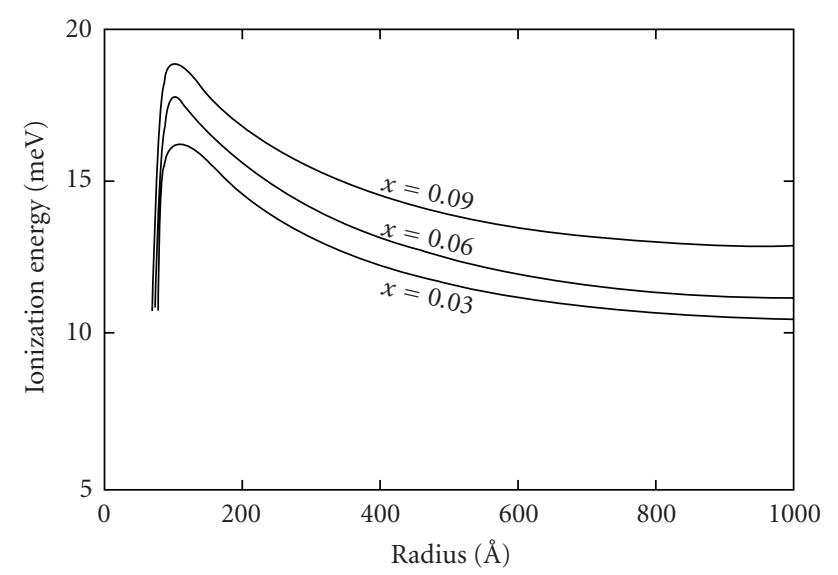

FIgURe 1: Variation of ionization energy with dot size for different concentrations of $\mathrm{Mn}$ ions in a $\mathrm{Cd}_{1-x} \mathrm{Mn}_{x} \mathrm{Te} \mathrm{QD}$.

the kinetic exchange contribution which, in principle, can induce spontaneous spin-polarization, that is, in the absence of an external magnetic field. Choosing the cell dimension in CdTe to be $6.48 \AA \grave{A}$, taking 8 atoms per unit cell we have calculated the total number of ions present in a spherical quantum dot for different concentrations. Thus the spin exchange interaction energy among $\mathrm{Mn}$ ions is obtained for two different dot radii as shown in Figure 4.

2.4. Magnetization Energy. Following formalism describes the magnetization energy in the CdMnTe dot. Intending to consider semimagnetic semiconductors $\mathrm{Cd}_{1-x_{\text {in }}} \mathrm{Mn}_{x_{\text {in }}} \mathrm{Te}$ we will incorporate into the Hamiltonian the exchange Heisenberg interaction of the conduction band electrons with $\mathrm{Mn}$ ions. We consider the magnetic Hamiltonian for one itinerant electron with spin $\bar{s}$ located at $\bar{r}$ and one Mn ions with $S_{n}^{\mathrm{Mn}}$ located at $R_{n}$ as

$$
H_{m}=-\sum_{n} J_{s \mathrm{~d}}\left(r-R_{n}\right) S_{n}^{\mathrm{Mn}} s
$$

where $J_{s-d}$ is the coupling strength due to the spin-spin exchange interaction between the $\mathrm{d}$ electrons of the $\mathrm{Mn}^{+2}$ cations and the s- or p-band electrons; it is negative for conduction band electrons and the sum runs over all the $\mathrm{Mn}$ ions. The value of $J_{\mathrm{sd}}$ is taken as $12 \times 10^{-3} \mathrm{eVnm}^{3}$. We will use the mean-field approximation inserting the mean value of $\mathrm{Mn}$ spin in $z$ direction. In the mean field approximation, $H^{e} \rightarrow H_{\mathrm{Mn}}^{e} \equiv g_{e} \mu_{B} \bar{s} \cdot \bar{h}^{e}$ and $H^{\mathrm{Mn}} \rightarrow H_{\mathrm{MF}}^{\mathrm{Mn}} \equiv g_{\mathrm{Mn}} \mu_{B} \bar{s} \cdot \bar{h}^{\mathrm{Mn}}$, where $g_{\mathrm{Mn}}=2, \mu_{B}$ is the Bohr magneton, $S=5 / 2$ is the spin of a manganese atom, and $h^{e}$ and $h^{\mathrm{Mn}}$ are the effective magnetic fields acting upon electrons and magnetic impurities, respectively, and can be given as

$$
\begin{aligned}
h^{e} & =\frac{1}{g_{e} \mu_{B}} J_{\mathrm{sd}} N_{\mathrm{Mn}}\left\langle S_{z}\right\rangle, \\
h^{\mathrm{Mn}} & =\frac{1}{g_{e} \mu_{B}} J_{\mathrm{sd}} M^{e} .
\end{aligned}
$$

Here, $N_{\mathrm{Mn}}=4 x / a_{o}^{3}$ is the density of Mn ions with $a_{o}^{3}$ being the unit cell volume. $M^{e}=\sum_{n} J_{\mathrm{sd}}\langle s\rangle \delta\left(r-R_{n}\right)$ is

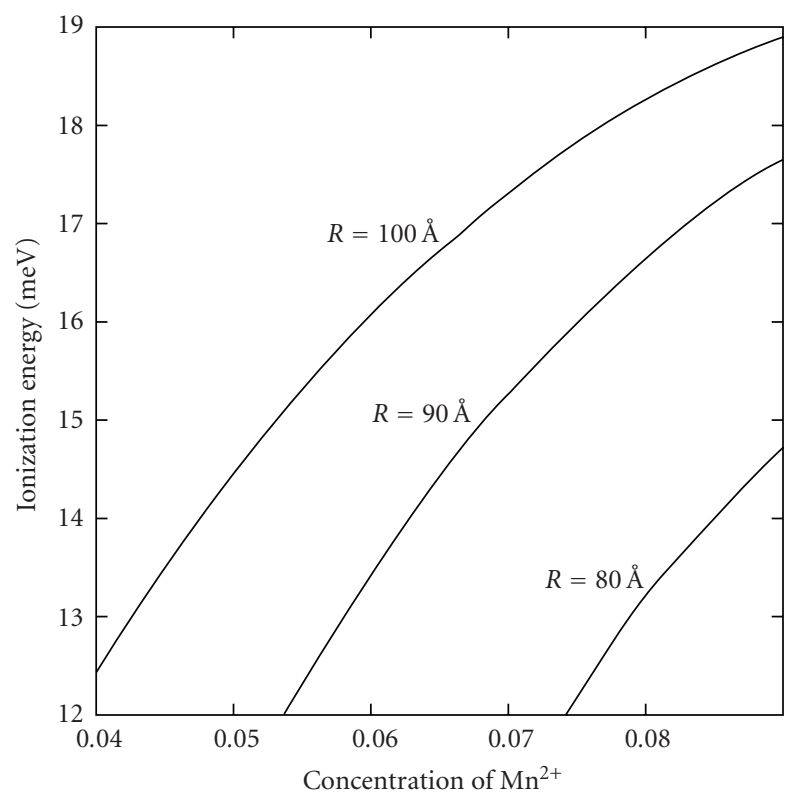

FIGURE 2: Variation of ionization energy with concentration of Mn ions for different sizes of dot of $\mathrm{Cd}_{1-x} \mathrm{Mn}_{x} \mathrm{Te}$.

the magnetization density of the electron subsystem which is assumed to be uniform within the length scale of the magnetic interactions; so the magnetic response of the Fermi sea electrons to the field $h^{e}$ is given by

$$
M^{e}=g_{e} \mu_{B} s^{2} D^{e}\left(E_{F}\right) h^{e},
$$

where the density of states of electron gas with the effective mass and the electron concentration $n$ is

$$
D^{e}\left(E_{F}\right)=\left(3 \pi^{2}\right)^{-2 / 3}\left(3 m^{*} / \hbar^{2}\right) n^{1 / 3} .
$$

On the other hand, the magnetic response of the impurity spin to the effective field $h^{\mathrm{Mn}}$ is given by

$M^{\mathrm{Mn}}=g_{\mathrm{Mn}} \mu_{B} N_{\mathrm{Mn}}\left\langle S_{z}\right\rangle=g_{\mathrm{Mn}} \mu_{B} N_{\mathrm{Mn}} S B_{s}\left(\frac{g_{\mathrm{Mn}} \mu_{B} N_{\mathrm{Mn}} S h^{\mathrm{Mn}}}{k_{B} T}\right)$,

where $B_{s}(x)$ is given as (10). So within the spirit of a mean field framework the magnetization of Mn subsystem is the given by

$$
M^{\mathrm{Mn}}=g_{\mathrm{Mn}} \mu_{B} N_{\mathrm{Mn}} S B_{s}\left(\frac{J_{\mathrm{sd}} S}{2 k_{B} T} M^{e}\right),
$$

which should be determined self consistently with the electron magnetization:

$$
M^{e}=\frac{J_{\mathrm{sd}} D^{e}\left(E_{F}\right)}{g_{\mathrm{Mn}} \mu_{B}} M^{\mathrm{Mn}} .
$$

\section{Results and Discussion}

The donor binding energy as a function of dot radius different concentrations of $\mathrm{Mn}$ ions is given in Figure 1. 


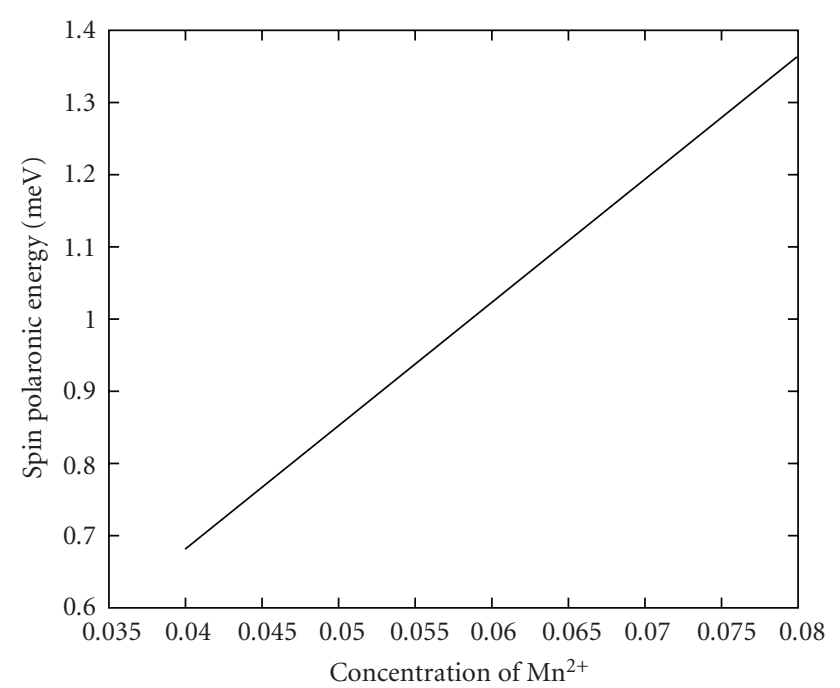

FIGURE 3: Variation of spin polaronic energy with concentration of Mn ions for the dot size of $100 \AA$.

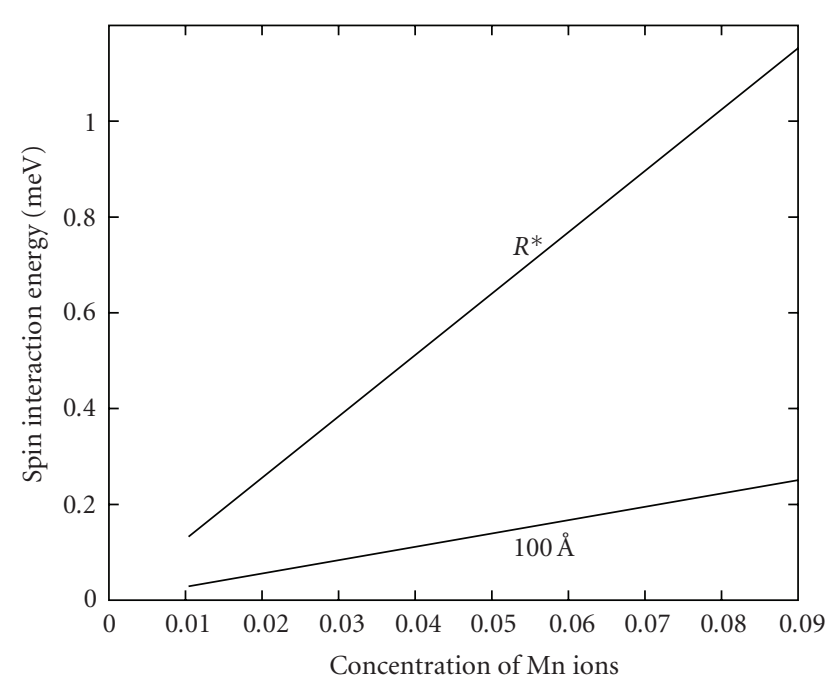

FIGURE 4: Variation of spin interaction energy with concentration of $\mathrm{Mn}$ ions for two different dot sizes of $\mathrm{Cd}_{1-x} \mathrm{Mn}_{x}$ Te QD. $\mathrm{R}^{*}=$ $60.08 \AA$ Á. The spin interaction energy has been calculated using (11) for different concentrations.

As expected, the binding energy decreases with an increase of dot radius, reaching the bulk value for larger dot radii. As the dot radius approaches zero, the confinement becomes negligibly small, and in the finite barrier problem the tunneling becomes huge. The binding energy again approaches the bulk value of the barrier. In all the cases, the ionization energy approaches the bulk value in both the limits of $L \rightarrow 0$ and $L \rightarrow \infty$ corresponding to one effective Bohr radius of CdTe, $11.38 \mathrm{meV}$, which is the ionization energy of the donor for the bulk. Hence the variation of ionization energy with dot radius shows a peak around $1.5 \mathrm{R}^{*}$ for all the concentrations. This is a well-known result in all quantum well structures [32]. Donor ionization

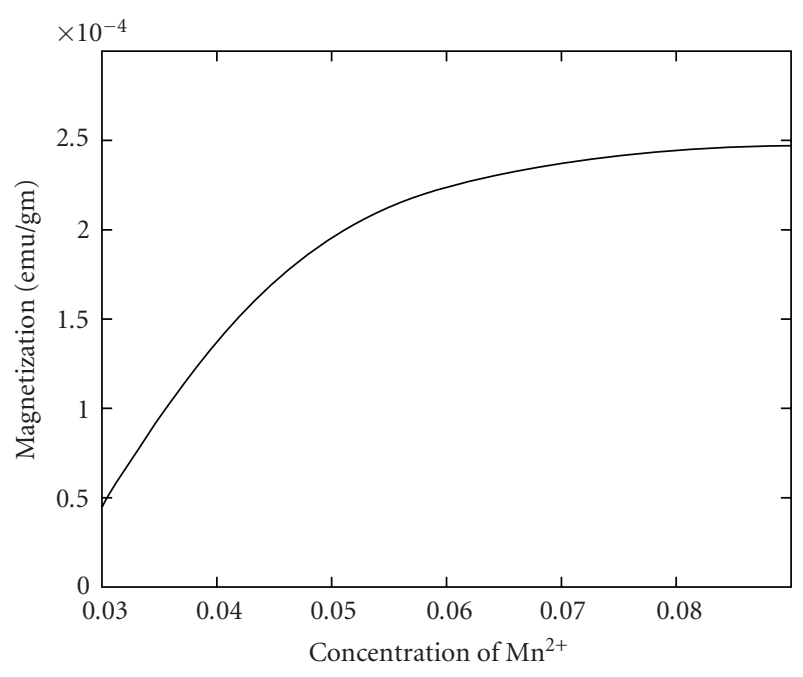

FIGURE 5: Variation of magnetization with different concentrations of $\mathrm{Mn}$ ions in a $\mathrm{Cd}_{1-x} \mathrm{Mn}_{x} \mathrm{Te} \mathrm{QD}$ with a finite barrier height.

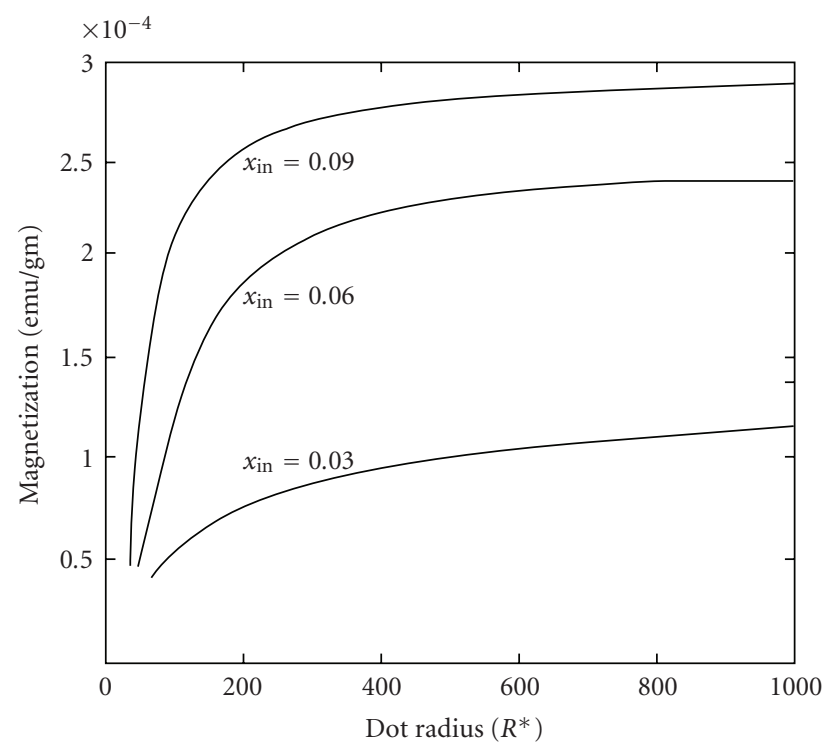

FIGURE 6: Variation of magnetization with dot radius for different concentrations of $\mathrm{Mn}$ ions in a $\mathrm{Cd}_{1-x_{\text {in }}} \mathrm{Mn}_{x_{\text {in }}} \mathrm{Te} / \mathrm{Cd}_{1-x_{\text {out }}} \mathrm{Mn}_{x_{\text {out }}} \mathrm{Te}$ QD.

energy becomes higher with the concentration for any dot radii.

Figure 2 represents the variation of ionization energies of a donor impurity with concentration of $\mathrm{Mn}$ ions for three different dot radii. It is clear that donor ionization energy increases with the concentration. For larger dot radii, the ionization energy increases slowly with the increase of concentration whereas the variation of increase in ionization energy is sharper for smaller dots due to the confinement. These results are very good agreement with the other investigators qualitatively $[33,34]$. The slope measures with the values of $133 \mathrm{meV} / \AA^{3}$ for $=100 \AA, 125 \mathrm{meV} / \AA^{3}$ for $90 \AA$ and $111 \mathrm{meV} / \AA^{3}$ for $80 \AA$. 
In Figure 3, we have plotted the variation of spin polaronic energy with concentration of $\mathrm{Mn}$ ions for the dot size of $100 \AA$ Á. The spin polarization energy increases with the concentration of $\mathrm{Mn}$ ions. This variation is found to be linearly varying. Our results are closely in agreement with the results of Kai Chang et al. [30], who have found the energy dispersion of an electron in a double quantum wire with a diluted magnetic semiconductor barrier recently.

Figure 4 shows the variation of spin interaction energy among $\mathrm{Mn}$ ions with for two different dot sizes of $\mathrm{Cd}_{1-x} \mathrm{Mn}_{x} \mathrm{Te} \mathrm{QD}$. In both cases the spin exchange interaction energy of confined electrons increases with the concentrations linearly. The variation of spin correlation energy is sharper when the radius of the dot is higher whereas there occurs a slow variation for smaller dot radii.

In Figure 5, we have plotted for the variation of magnetization with different concentrations of $\mathrm{Mn}$ ions in a $\mathrm{Cd}_{1-x_{\text {in }}} \mathrm{Mn}_{x_{\text {in }}} \mathrm{Te} / \mathrm{Cd}_{1-x_{\text {out }}} \mathrm{Mn}_{x_{\text {out }}} \mathrm{Te} \mathrm{QD}$. As the concentration of $\mathrm{Mn}$ ions increases, the magnetization of Mn subsystem also increases. This mechanism has clearly brought out in Figure 6 which has been plotted for variation of magnetization with dot radius for different concentrations of $\mathrm{Mn}$ ions in a $\mathrm{Cd}_{1-x_{\text {in }}} \mathrm{Mn}_{x_{\text {in }}} \mathrm{Te} / \mathrm{Cd}_{1-x_{\text {out }}} \mathrm{Mn}_{x_{\text {out }}} \mathrm{Te}$ in a finite barrier model. Magnetization decreases when dot radius increases for all the concentrations of $\mathrm{Mn}$ ions. As concentration increases, the magnetization also increases for all the dot radii whereas these changes occur appreciably for smaller dots. Magnetization is stronger for smaller dots with high concentration as expected. Magnetization is almost constant for larger dot radii for any concentration of $\mathrm{Mn}$ ions [35]. Magnetization of the magnetic ions competes with spin-orbit coupling, and the effects of the spin-subband populations and the spin-polarization as functions of the temperature, $T$, and the in-plane magnetic field, $B$, for narrow to wide dilutemagnetic-semiconductor quantum wells have been studied recently [35].

In conclusion, the spin polarization energy of a confined donor electron has been studied for different concentrations of $\mathrm{Mn}$ ions for the finite dot of $\mathrm{Cd}_{1-x} \mathrm{Mn}_{x} \mathrm{Te}$. The magnetization of Mn subsystems and the strength of spin exchange energy of confined electrons have been discussed. The main results are the spin polaronic effect raises the binding energy, but this feature predominantly occurs only for smaller dots, the variation of increase in ionization energy is sharper for smaller dots due to the confinement, and the spin interaction energy increases with the concentration of $\mathrm{Mn}$ ions having a sharp variation for larger dots. However, this problem may be improved in the line of thought of considering spin polarization energy self consistently which requires a lot of computation techniques. Experimental efforts are encouraged to lend support to our calculations.

\section{References}

[1] S. Baskoutas, "Excitons and charged excitons in InAs nanorods," Chemical Physics Letters, vol. 404, no. 1-3, pp. 107111, 2005.

[2] T. Mizokawa, T. Nambu, A. Fujimori, T. Fukumura, and M. Kawasaki, "Electronic structure of the oxide-diluted magnetic semiconductor $\mathrm{Zn}_{1-x} \mathrm{Mn}_{x} \mathrm{O}$," Physical Review B, vol. 65, no. 8, Article ID 085209, 5 pages, 2002.

[3] D. D. Awschalom and M. E. Flatté, "Challenges for semiconductor spintronics," Nature Physics, vol. 3, no. 3, pp. 153-159, 2007.

[4] R. J. Soulen Jr., J. M. Byers, M. S. Osofsky, et al., "Measuring the spin polarization of a metal with a superconducting point contact," Science, vol. 282, no. 5386, pp. 85-88, 1998.

[5] S. K. Upadhyay, A. Palanisami, R. N. Louie, and R. A. Buhrman, "Probing ferromagnets with andreev reflection," Physical Review Letters, vol. 81, no. 15, pp. 3247-3250, 1998.

[6] Y. Ji, G. J. Strijkers, F. Y. Yang, et al., "Determination of the spin polarization of half-metallic $\mathrm{CrO}_{2}$ by point contact Andreev reflection," Physical Review Letters, vol. 86, no. 24, pp. 55855588, 2001.

[7] G. J. Strijkers, Y. Ji, F. Y. Yang, C. L. Chien, and J. M. Byers, "Andreev reflections at metal/superconductor point contacts: measurement and analysis," Physical Review B, vol. 63, no. 10, Article ID 104510, 6 pages, 2001.

[8] J. G. Braden, J. S. Parker, P. Xiong, S. H. Chun, and N. Samarth, "Direct measurement of the spin polarization of the magnetic semiconductor (Ga,Mn)As," Physical Review Letters, vol. 91, no. 5, Article ID 056602, 4 pages, 2003.

[9] R. P. Panguluri, B. Nadgorny, T. Wojtowicz, W. L. Lim, X. Liu, and J. K. Furdyna, "Measurement of spin polarization by Andreev reflection in ferromagnetic $\mathrm{In}_{1-x} \mathrm{Mn}_{x} \mathrm{Sb}$ epilayers," Applied Physics Letters, vol. 84, no. 24, pp. 4947-4949, 2004.

[10] R. P. Panguluri, B. Nadgorny, T. Wojtowicz, X. Liu, and J. K. Furdyna, "Inelastic scattering and spin polarization in dilute magnetic semiconductor (Ga,Mn)Sb," Applied Physics Letters, vol. 91, no. 25, Article ID 252502, 3 pages, 2007.

[11] H. Ohno, "Making nonmagnetic semiconductors ferromagnetic," Science, vol. 281, no. 5379, pp. 951-956, 1998.

[12] T. Jungwirth, J. Sinova, J. Mašek, J. Kučera, and A. H. MacDonald, "Theory of ferromagnetic (III,Mn)V semiconductors," Reviews of Modern Physics, vol. 78, no. 3, Article ID 809, 56 pages, 2006.

[13] L. M. Smith, T. Gurung, S. Mackowski, H. E. Jackson, G. Karczewski, and J. Kossut, "Magnetization imaging of single CdMnTe/ZnTe quantum dots," in Proceedings of Quantum Electronics and Laser Science Conference (QELS '05), vol. 2, pp. 1059-1060, Baltimore, Md, USA, May 2005.

[14] A. Haury, A. Wasiela, A. Arnoult, et al., "Observation of a ferromagnetic transition induced by two-dimensional hole gas in modulation-doped CdMnTe quantum wells," Physical Review Letters, vol. 79, no. 3, pp. 511-514, 1997.

[15] M. Tanaka, "Epitaxial growth and properties of III-V magnetic semiconductor (GaMn)As and its heterostructures," Journal of Vacuum Science and Technology B, vol. 16, no. 4, pp. 22672274, 1998.

[16] R. M. Abolfath, A. G. Petukhov, and I. Žutić, "Piezomagnetic quantum dots," Physical Review Letters, vol. 101, no. 20, Article ID 207202, 4 pages, 2008.

[17] R. M. Abolfath, P. Hawrylak, and I. Žutić, "Tailoring magnetism in quantum dots," Physical Review Letters, vol. 98, no. 20, Article ID 207203, 4 pages, 2007.

[18] I. Žutić, J. Fabian, and S. D. Sarma, "Spintronics: fundamentals and applications," Reviews of Modern Physics, vol. 76, no. 2, pp. 323-410, 2004.

[19] A. G. Petukhov, I. Zutić, and S. C. Erwin, "Thermodynamics of carrier-mediated magnetism in semiconductors," Physical Review Letters, vol. 99, no. 25, Article ID 257202, 4 pages, 2007.

[20] J. K. Furdyna, "Diluted magnetic semiconductors," Journal of Applied Physics, vol. 64, no. 4, pp. R29-R64, 1988. 
[21] A. A. Dremin, D. R. Yakovlev, A. A. Sirenko, et al., "Electron cyclotron mass in undoped CdTe/CdMnTe quantum wells," Physical Review B, vol. 72, no. 19, Article ID 195337, 5 pages, 2005.

[22] T. Fukumura, H. Toyosaki, and Y. Yamada, "Magnetic oxide semiconductors," Semiconductor Science and Technology, vol. 20, no. 4, pp. S103-S111, 2005.

[23] T. Fukumura, Y. Yamada, H. Toyosaki, T. Hasegawa, H. Koinuma, and M. Kawasaki, "Exploration of oxide-based diluted magnetic semiconductors toward transparent spintronics," Applied Surface Science, vol. 223, no. 1-3, pp. 62-67, 2004.

[24] N. Takahashi, K. Takabayashi, I. Souma, J. Shen, and Y. Oka, "Magnetoluminescence in quantum dots and quantum wires of II-VI diluted magnetic semiconductors," Journal of Applied Physics, vol. 87, no. 9, pp. 6469-6471, 2000.

[25] I. P. Smorchkova, F. S. Flack, N. Samarth, J. M. Kikkawa, S. A. Crooker, and D. D. Awschalom, "Spin transport and optically-probed coherence in magnetic semiconductor heterostructures," Physica B, vol. 249-251, pp. 676-684, 1998.

[26] T. Kasuya and A. Yanase, "Anomalous transport phenomena in eu-chalcogenide alloys," Reviews of Modern Physics, vol. 40, no. 4, pp. 684-696, 1968.

[27] J. A. Gaj, R. Planel, and G. Fishman, "Relation of magnetooptical properties of free excitons to spin alignment of $\mathrm{Mn}^{2+}$ ions in $\mathrm{Cd}_{1-x} \mathrm{Mn}_{x}$ Te," Solid State Communications, vol. 29, no. 5, pp. 435-438, 1979.

[28] T. Hayashi, M. Tanaka, and A. Asamitsu, "Tunneling magnetoresistance of a GaMnAs-based double barrier ferromagnetic tunnel junction," Journal of Applied Physics, vol. 87, no. 9, pp. 4673-4675, 2000.

[29] K. Gnanasekar and K. Navaneethakrishnan, "Spin polaron in a quantum dot of the diluted magnetic semiconductors," Modern Physics Letters B, vol. 18, no. 10, pp. 419-426, 2004.

[30] K. Chang and F. M. Peeters, "Spin-polarized ballistic transport in diluted magnetic semiconductor quantum wire systems," Physical Review B, vol. 68, no. 20, Article ID 205320, 5 pages, 2003.

[31] C. Simserides, "Spin-subband populations and spin polarization of quasi-two-dimensional carriers under an in-plane magnetic field," Physical Review B, vol. 75, no. 19, Article ID 195344, 7 pages, 2007.

[32] Sr. Gerardin Jayam and K. Navaneethakrishnan, "Optical properties of acceptors in semimagnetic quantum well systems," International Journal of Modern Physics B, vol. 16, no. 25, pp. 3737-3757, 2002.

[33] G. Mackh, W. Ossau, D. R. Yakovlev, et al., "Localized exciton magnetic polarons in $\mathrm{Cd}_{1-x} \mathrm{Mn}_{x}$ Te," Physical Review B, vol. 49, no. 15, pp. 10248-10258, 1994.

[34] A. O. Govorov, "Optical probing of the spin state of a single magnetic impurity in a self-assembled quantum dot," Physical Review B, vol. 70, no. 3, Article ID 035321, 5 pages, 2004.

[35] C. Simserides and I. Galanakis, "Quasi-two-dimensional carriers in dilute-magnetic-semiconductor quantum wells under in-plane magnetic field," Physica E, vol. 40, no. 5, pp. 12141216, 2008. 

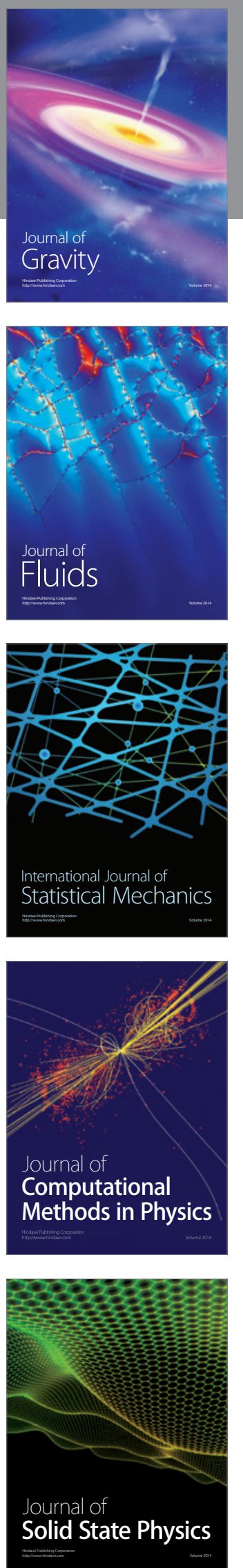

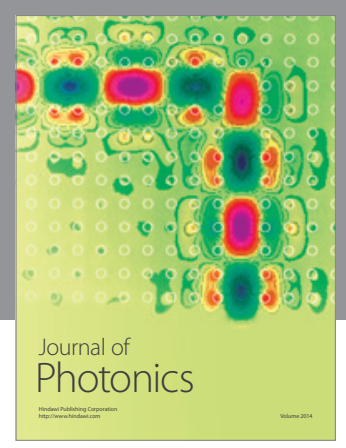

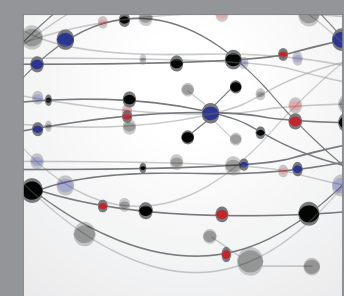

The Scientific World Journal
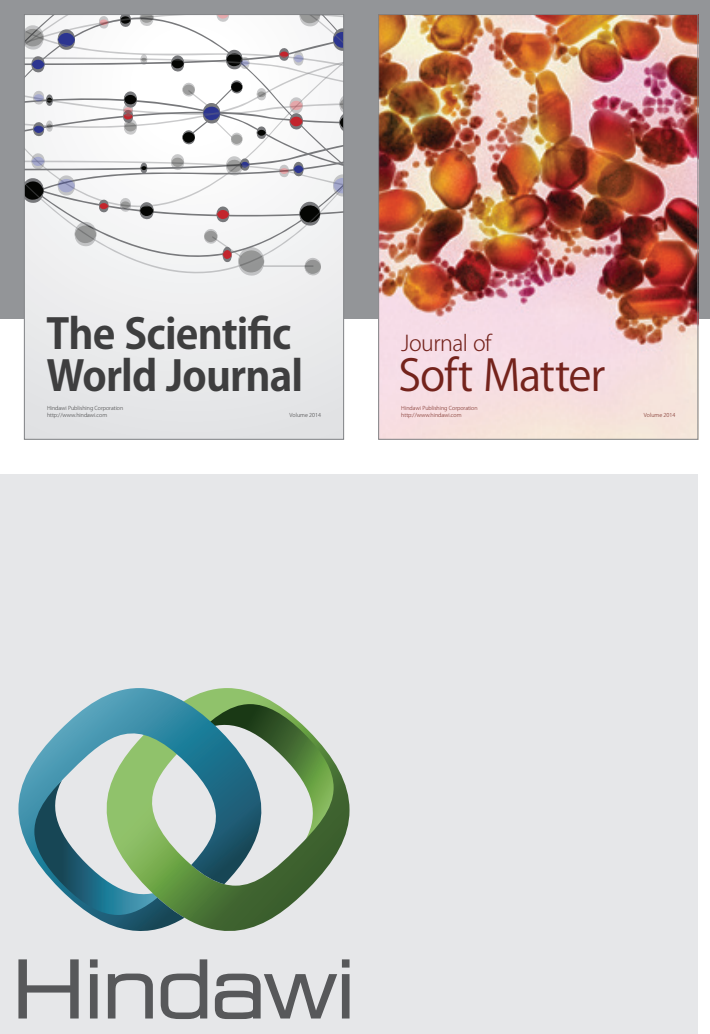

Submit your manuscripts at

http://www.hindawi.com
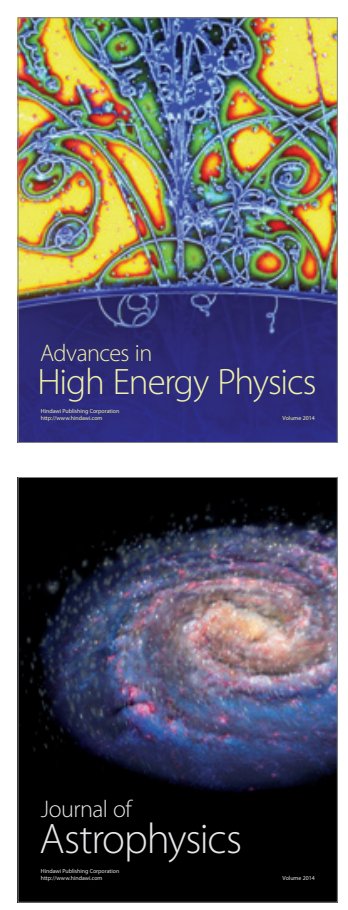
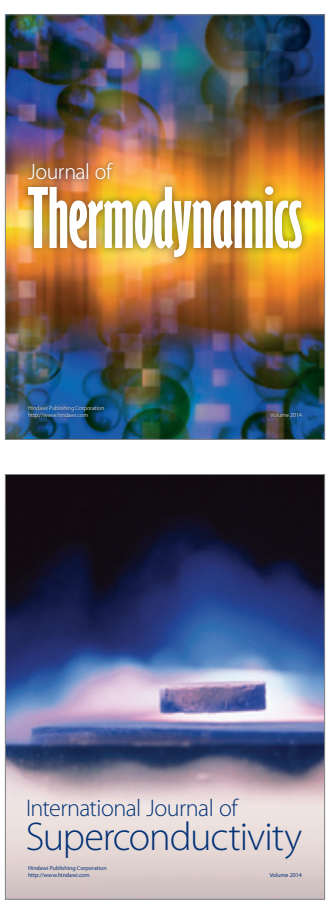
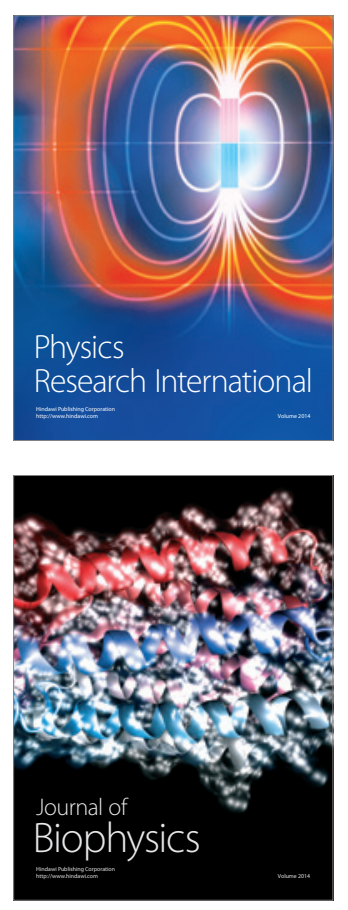
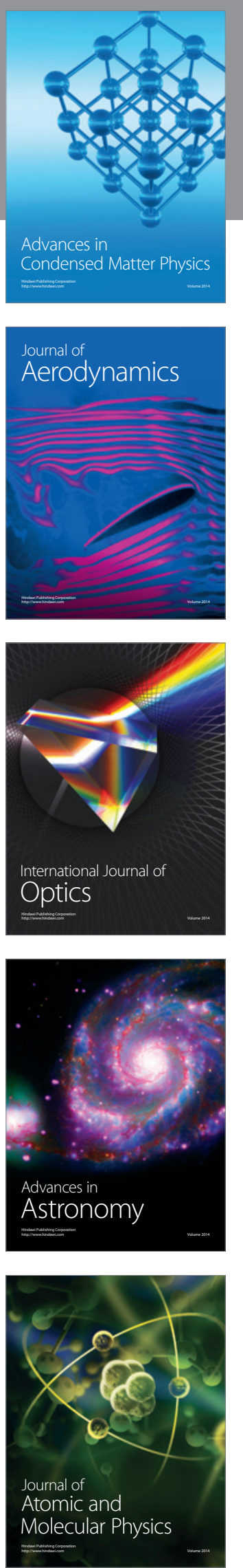